\title{
CARACTERIZAÇÃO DA PRÁTICA DE AUTOMEDICAÇÃO COM ANALGÉSICOS PARA O TRATAMENTO DA DOR
}

Cristiane Martinez Ruiz Pegoraro, Renata Mano Scatamburlo Bifaroni, Edson Asssunção Mareco, Thaise Ramires Tonizza, Ludmila Ichioka Silgueiro

Universidade do Oeste Paulista - UNOESTE, Presidente Prudente, SP. E-mail: cristiane@unoeste.br

\section{RESUMO}

A automedicação é muito comum entre pessoas de todas as idades, geralmente ocorre por indicações de terceiros, por conhecimento próprio ou fácil acesso em farmácias. O objetivo do estudo foi avaliar e verificar a prevalência de automedicação para o tratamento da dor em pacientes atendidos em campanhas de saúde por uma Universidade de Presidente Prudente. Aplicou-se um questionário envolvendo aspectos sócio-demográficos, uso de medicamento, a prática de automedicação e os tipos de dor. A amostra consistiu de 248 participantes, onde 203 admitiram a utilização de anti-inflamatórios não esteroides (AINEs). Destes 203, $166(81,7 \%)$ relataram automedicação, sendo dipirona $(48,5 \%)$ e paracetamol $(21,1 \%)$, os AINEs mais mencionados. A dor de cabeça foi mais prevalente, em mulheres $99(26,8 \%)$ e homens 51 $(13,8 \%)$, sendo as mulheres consideradas as que mais leem a bula dos medicamentos $70(34,5 \%)$ e conhecem os efeitos adversos dos mesmos 49 (24,2\%). Pode-se concluir que a prática da automedicação é muito frequente na população estudada.

Palavras-chave: AINES, automedicação, dores, efeitos colaterais, população.

\section{CHARACTERIZATION OF THE AUTOMEDICATION PRACTICE WITH ANALGESICS FOR THE PAIN TREATMENT}

\begin{abstract}
Self-medication is very common among people of all ages, usually through nominations from third parties, self-knowledge or easy access in pharmacies. The objective of the study was to evaluate and verify the prevalence of self-medication for the treatment of pain in patients seen in campaigns conducted by Universidade do Oeste Paulista. A questionnaire was applied involving socio-demographic aspects, drug use, the practice of self-medication and types of pain. The sample consisted of 248 participants, where 203 admitted the use of non-steroidal anti-inflammatory drugs (AINEs). Of these 203, 166 (81.7\%) reported selfmedication, being dipyrone (48.5\%) and paracetamol (21.1\%), the most frequently mentioned AINEs. The headache was more prevalent in women $99(26.8 \%)$ and men $51(13.8 \%)$, being women considered the ones who most read the package leaflet $70(34.5 \%)$ and know the effects adverse effects of the same 49 (24.2\%). It can be concluded that the practice of self-medication is very frequent in the studied population. Keywords: AINEs, self medication, pain, side effects, population.
\end{abstract}

\section{INTRODUÇÃO}

A automedicação é uma prática muito comum entre pessoas de todas as idades, normalmente acontece o uso de medicamentos sem prescrição médica, usualmente por indicações de terceiros ou por próprio conhecimento e/ou pelo fácil acesso aos medicamentos em farmácias devido alguns constarem na lista de Medicamentos Isentos de Prescrição (MIP) ${ }^{1}$.

Segundo a Organização Mundial de Saúde e o Ministério da Saúde, o mercado brasileiro dispõe de mais de 32 mil medicamentos que não poderiam ser vendidos 
sem a apresentação da receita, porém esta é dispensada de forma indiscriminada pelas farmácias favorecendo a automedicação ${ }^{2}$.

Os anti-inflamatórios não esteroides

$\left(A I N E_{S}\right)$ são os medicamentos mais utilizados mundialmente, frequentemente prescritos para alívio de dores e inflamação por apresentarem propriedades analgésicas, antipiréticas e antiinflamatórias. Os $\mathrm{AINE}_{S}$ inibem as enzimas COX (cicloxigenases) e a produção de prostanóides (tromboxano, prostaciclina e prostaglandinas), seus principais efeitos terapêuticos derivam da sua capacidade de inibir a produção de prostaglandinas derivadas da COX-2 que é induzível na grande maioria dos tecidos ${ }^{3}$.

A utilização desses medicamentos pode muitas vezes esconder uma doença, agravar novos problemas de saúde, causar efeitos adversos e interações medicamentosas, prejudicando a saúde do paciente. Os sintomas mais comuns associados a esses fármacos são gastrintestinais, incluindo anorexia, náuseas, dispepsia, dor abdominal e diarreia, podendo estar relacionados com a indução de úlceras gástricas ou intestinais ${ }^{3}$.

A população, muitas vezes, não tem acesso a informações completas a respeito da segurança dos fármacos. A maior parte das pessoas sequer conhece $O$ conjunto dos possíveis efeitos nocivos que a automedicação pode trazer, ou não sabe identificar nem prevenir corretamente combinações perigosas entre as substâncias farmacológicas. Por outro lado, alguns pacientes ignoram os perigos da associação de medicamentos, sua segurança fica em risco, com isso a presença do farmacêutico torna-se importante para prestar orientação do uso de qualquer medicamento e garantir um uso racional dos mesmos ${ }^{4}$.

O objetivo do estudo foi verificar a prevalência da automedicação para o tratamento da dor em pessoas atendidas em campanhas de saúde realizadas por uma Universidade de Presidente Prudente, determinando as principais dores que levam ao uso dos AINES; identificar os representantes mais consumidos dessa classe farmacológica, a frequência do uso deste fármaco e a forma de obtenção do mesmo e verificar o conhecimento da população sobre os riscos da automedicação.

\section{METODOLOGIA}

Durante a realização de uma Campanha de Saúde em cinco de maio de 2017, no calçadão de uma Universidade e no calçadão do centro da cidade, por meio de um projeto de extensão cadastrado sob no 6648/2017, os estudantes participantes da extensão (previamente capacitados) aplicaram um questionário para coleta de dados.

Os pesquisados foram abordados aleatoriamente durante a realização da campanha independente do sexo, idade ou outro fator qualquer e esclarecidos sobre a existência da pesquisa e a que se referia. A coleta dos dados foi realizada no primeiro semestre de 2017.

O questionário foi elaborado de acordo com Pilger et al. ${ }^{5}$ e Silva et al. ${ }^{6}$. O projeto foi aprovado no comitê de ética em pesquisa - CEP (CAAE: 70650317.0.0000.5515, parecer: 073519/2017). No questionário não constava identificação do pesquisado e o termo de consentimento livre e esclarecido (TCLE) foi dispensado pelo CEP. Composto por treze perguntas que abordavam aspectos sócio demográficos (idade, sexo, ocupação, estado civil, nível de escolaridade e renda familiar), o uso de medicamento e a prática de automedicação (frequência de uso, quem o recomendou, conhecimento sobre as possíveis reações adversas e leitura da bula,) e por fim perguntas relacionadas a dor (tipo de dor, intensidade e frequência).

A análise estatística foi realizada utilizando ambiente de programação $R$ (versão 3.3.2). Para as análises, foram utilizados os pacotes estatísticos fisher.test e chisq.test, seguido do teste de associação de cramer'sV (biblioteca - Isr).

\section{RESULTADOS}

Participaram do estudo 248 aleatoriamente nos calçadões de uma universidade e numa praça pública de Presidente Prudente, em sua maioria do sexo feminino 129 (52\%), sendo menos prevalente indivíduos do sexo masculino 119 (48\%). Quanto ao grau de escolaridade, notou-se que a maioria dos indivíduos possuía ensino superior incompleto. Em relação à renda familiar, prevaleceu a faixa entre 2 a 5 salários mínimos (um salário mínimo correspondia a $\mathrm{R} \$$ $1.108,38)$.

Dentre os 248 participantes, 45 não faziam uso de medicamentos para dor sendo $35(14,2 \%)$ do sexo masculino e $10(4,0 \%)$ do 
sexo feminino; já os 203 restantes, 84 (33,9\%) do sexo masculino e 119 (47,9\%) do sexo feminino afirmaram fazer uso destes fármacos. O estudo prosseguiu com os 203 participantes que relataram fazer uso destes medicamentos ( $41,4 \%$ do sexo masculino e $58,6 \%$ do sexo feminino), sendo o sexo feminino que faz maior utilização de medicamentos para a dor $(p<0,001)$.

Quanto às formas de aquisição dos medicamentos, $15(7,4 \%)$ do sexo masculino e $22(10,9 \%)$ do sexo feminino foram por prescrição e 69 (33,9\%) do sexo masculino e 97 $(47,8 \%)$ do sexo feminino foram por automedicação. Para estes dados não houve diferenças estatísticas.

Ao serem questionados sobre a frequência com que faziam uso dos medicamentos $6(2,9 \%)$ dos participantes do sexo masculino citaram que 0 fazem diariamente, $2(0,9 \%)$ mais de 3 vezes por semana, 16 (7,9\%) semanalmente, 34 (16,7\%) ocasionalmente e $26(12,8 \%)$ raramente, já os participantes do sexo feminino $11(5,4 \%)$ disseram que o fazem diariamente, $7(3,4 \%)$ mais de 3 vezes por semana, 27 (13,3\%) semanalmente, $57(28,1 \%)$ ocasionalmente e $17(8,4 \%)$ raramente. Para estes dados não houve diferenças estatísticas.

Com relação à ocorrência da dor que os levou a utilizar esses medicamentos, os participantes puderam escolher nove opções diferentes na mesma pergunta (número cinco) que constava no questionário (com isso as respostas estão acima da quantidade de participantes). Para o sexo masculino tivemos 51 participantes $(13,8 \%)$ que apresentaram dor de cabeça, $17(4,6 \%)$ dor nas costas, $5(1,4 \%)$ dor de dente, $9(2,4 \%)$ dor de estômago, 12 $(3,3 \%)$ dor de garganta e $27(7,3 \%)$ dor muscular. Já para o sexo feminino tivemos 99 participantes $(26,8 \%)$ que apresentaram dor de cabeça, $35(9,5 \%)$ dor nas costas, $3(0,8 \%)$ dor de dente, $20(5,4 \%)$ dor de estômago, 21 $(5,7 \%)$ dor de garganta e $38(10,3 \%)$ dor muscular. As outras tomadas $10(2,8 \%)$ para o sexo masculino e $22(5,9 \%)$ para o feminino foram representadas por dores no pescoço, ombros, braços, pernas, pés, joelhos, cólicas renais, artrose, febres e alergias. Para estes dados não houve diferenças estatísticas. Observou-se grande uso de medicamentos para dor por cólicas menstruais, no grupo do sexo feminino.

Com relação aos medicamentos que os participantes relataram utilizar, o grupo dos AINES foi o mais prevalente, seguido dos relaxantes musculares e outros como escopolamina, omeprazol, maleato de clorfeniramina com cloridrato de fenilefrina, azitromicina, amoxicilina, clonazepam, tramadol, ciclobenzaprina, naratriptano, simeticona e amitriptilina. Figura 1.

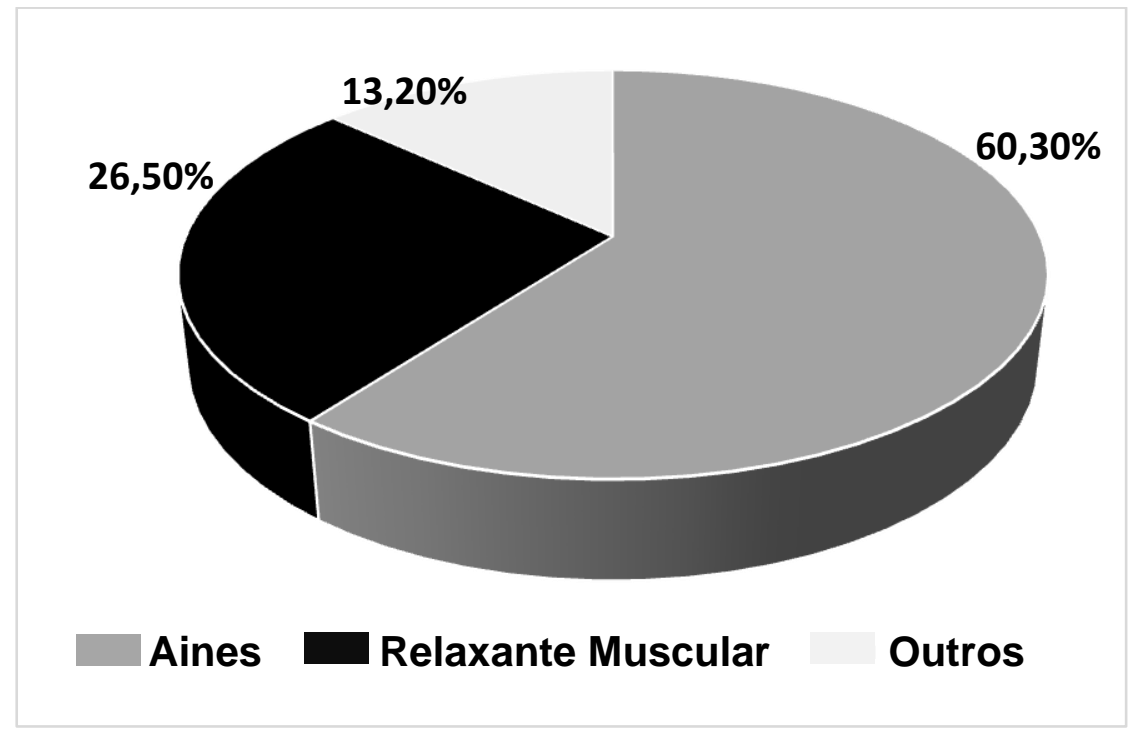

Figura 1. Prevalência dos grupos de medicamentos mais consumidos.

Nessa mesma pergunta (número 4 do questionário) o participante poderia citar diversos fármacos, obtendo-se assim valores maiores do que a quantidade de pessoas 
participantes. Entre os AINEs mais utilizados pelos participantes obtivemos a dipirona $62,1 \%$ para o sexo feminino e $37,9 \%$ para o masculino; o paracetamol $34 \%$ para o feminino e $66 \%$ para o masculino; a nimesulida $54,5 \%$ para o feminino e $45,5 \%$ para o masculino; o Ibuprofeno $81,9 \%$ para o feminino e $18,1 \%$ para o masculino e o diclofenaco que só foi utilizado apenas pelo sexo masculino (100\%). Havendo diferença estatística entre os sexos quanto ao medicamento mais consumido, prevalecendo o sexo feminino que utiliza mais dipirona, nimesulida e ibuprofeno e no sexo masculino prevaleceram os medicamentos paracetamol e diclofenaco, $p<0,001$.

Dentre as justificativas para a realização da automedicação, as mais relatadas pelos participantes foram: utilização do fármaco anteriormente já prescrito pelo médico $(12,3 \%$ sexo masculino e $15,3 \%$ sexo feminino), indicações de familiares, amigos ou pessoas conhecidas $(10,8 \%$ do sexo masculino e $11,3 \%$ sexo feminino), facilidade de acesso ao medicamento $(4,9 \%$ sexo masculino e $8,9 \%$ sexo feminino), autoconhecimento $(4,4 \%$ sexo masculino e $4,4 \%$ sexo feminino), indicação do farmacêutico ( $4,9 \%$ sexo masculino e $4,5 \%$ sexo feminino), propagandas apenas o sexo feminino $(1,0 \%)$ e outros motivos não relatados $(0,5 \%$ sexo masculino e $3,0 \%$ sexo feminino).

Quando questionado se os participantes sabiam dos efeitos colaterais desses medicamentos, 62 participantes (30,5\%) do sexo masculino e $70(34,5 \%)$ do sexo feminino afirmaram que não conheciam, já 22 $(10,8 \%)$ do sexo masculino e $49(24,2 \%)$ do sexo feminino descreveram ter conhecimento das reações adversas. Para esses dados houve diferença estatística $p<0,05$.

Quanto ao fato de ler a bula dos medicamentos apenas 31 dos participantes $(15,3 \%)$ do sexo masculino e $70(34,5 \%)$ do sexo feminino afirmaram ter o hábito de fazer essa leitura. Para esses dados houve diferença estatística $p<0,01$.

Com relação à orientação do farmacêutico, 103 (50,8\%) dos participantes afirmaram não receber orientações desse profissional, sendo $45(22,2 \%)$ do sexo masculino e $58(28,5 \%)$ do feminino; já 39 $(19,2 \%)$ do sexo masculino e $61(30,1 \%)$ do sexo feminino relataram receber orientações adequadas desses profissionais. Para esses dados não houve diferença estatística.

\section{DISCUSSÃO}

Foi percebido no presente estudo que o sexo feminino faz mais uso de medicamentos que o sexo masculino, isto pode estar relacionado ao fato das mulheres usarem mais medicamentos em inúmeras fases da vida por problemas de saúde com maior frequência, como cólicas menstruais e enxaqueca, além de possuírem maior preocupação com a sua saúde e historicamente são responsáveis pelos cuidados da saúde de sua família.

Quanto aos dados relacionados à aquisição de medicamentos por prescrição médica, foi observado que os homens são mais conscientes do que as mulheres. No estudo realizado por Silva et al. $^{2}$ que avaliaram a prevalência da automedicação em um centro de saúde universitário, 59,3 \% dos homens se automedicavam, não diferindo muito do sexo feminino, cuja porcentagem de pessoas que realizavam tal prática foi de $66,5 \%$. Já em outra pesquisa realizada por Almeida-Junior et al. ${ }^{8}$, a automedicação está representada por $54,46 \%$ de mulheres e $45,54 \%$ de homens.

Tanto no Brasil como em outros países a automedicação é uma prática amplamente difundida, assim como, a prescrição errônea pode acarretar efeitos indesejáveis, como o mascaramento de sintomas e doenças até o surgimento de enfermidades iatrogênicas, ou seja, doenças desencadeadas pelo uso incorreto do medicamento ${ }^{9}$.

A automedicação mostra-se ainda frequente na sociedade devido a uma soma de fatores, como a dificuldade de acesso, a demora e a baixa qualidade do atendimento nos serviços de saúde (tanto no setor público quanto no privado), a veiculação de propagandas de medicamentos isentos de prescrição na mídia, a presença da chamada "farmacinha caseira" nos domicílios e a crença da população de que os medicamentos resolvem todas as enfermidades ${ }^{10}$.

Quanto à frequência com que faziam uso do medicamento, observou-se que a maioria respondeu ocasionalmente, o que mostra que a população em geral tem um consentimento quanto à automedicação e demostra de maneira singular que tem um controle quanto ao seu uso, valores diferentes foram encontrados no estudo de Rankel et al. ${ }^{1}$, os quais $75 \%$ dos entrevistados afirmaram usar 
raramente medicamentos e no estudo de Almeida-Junior et al. $^{8} 56,50 \%$ declararam que fazem uso frequentemente e $18,50 \%$ raramente.

Com relação às dores que levaram a automedicação, a mais prevalente foi à dor de cabeça seguido de dor muscular. Nos estudos de Karyna et al. ${ }^{11}$, Fontanella et al. ${ }^{12} \mathrm{e}$ Lopes et al. ${ }^{13}$ encontrou-se resultados parecidos sendo a dor de cabeça a mais prevalente com $37 \%, 33,3 \%$ e $89,29 \%$, respectivamente.

A dor de cabeça sendo um dos principais motivos para automedicação, pode indicar um problema de saúde mais sério no qual carece de cuidados específicos, sendo assim é necessário que o profissional farmacêutico esteja atento às queixas dos seus pacientes, promovendo um uso racional da medicação e o aconselhamento quanto à necessidade de se buscar um profissional especializado para o correto tratamento da enfermidade ${ }^{13}$.

No presente estudo, os (AINEs), foram os medicamentos de maior prevalência de consumo. Resultado semelhante foi encontrado no estudo de Rankel et al. ${ }^{1}$ no qual $75 \%$ dos entrevistados afirmaram fazer uso de AINEs.

Os AINEs não seletivos quando usados de maneira irracional podem causar vários efeitos colaterais, sendo os mais comuns os efeitos gastrointestinais (úlceras gástricas e gastrites), isso se deve a inibição das ciclooxigenases e consequentemente das prostaglandinas cuja ação fisiológica é estimular a produção de muco e bicarbonato nas células epiteliais gástricas e redução da produção do ácido clorídrico nas células parietais ${ }^{3}$. Os AINES causam também uma redução das prostaglandinas renais que são vasodilatadoras nas arteríolas aferentes, consequentemente diminuirá a taxa de filtração glomerular com ativação do sistema renina angiotensina aldosterona provocando aumento da pressão arterial e risco de insuficiência renal ${ }^{14}$. Outros efeitos colaterais podem ser representados por aumento do tempo de sangramento (redução da formação do tromboxano), reações de hipersensibilidade, desvio metabólico para a via da lipooxigenase aumentando a formação dos leucotrienos que promovem broncoconstrição ${ }^{15}$.

Os medicamentos isentos de prescrição (MIP) por serem de fácil acesso à população e não precisarem de prescrição médica, podem ocasionar danos à saúde como qualquer outro tipo de medicamento quando usados de forma incorreta não respeitando a dose, posologia e o uso terapêutico ${ }^{16}$. No presente estudo, a dipirona e o ibuprofeno foram os mais utilizados pelo sexo feminino, já o paracetamol e o diclofenaco pelo masculino; apesar da nimesulida não ser isento de prescrição dentre os medicamentos de prevalência é o terceiro mais utilizado em ambos os sexos.

A dipirona pode causar algumas reações adversas como induzir a discrasia sanguínea, em especial a agranulocitose, porém estudos demonstram que os riscos da dipirona são similares ou menores do que o de outros analgésicos-antipiréticos sendo a ocorrência de aplasia medular rara no uso intensivo de dipirona ${ }^{17,18,19}$.

O paracetamol assim como a dipirona pode causar reações adversas se utilizado de maneira irracional, destacando-se seus efeitos hepatotóxicos que são comuns quando o mesmo é utilizado em doses elevadas ou continuadamente ${ }^{15}$.

A maioria dos participantes da pesquisa relatou não conhecer os efeitos adversos dos medicamentos. A prevalência das mulheres deve-se ao fato de provavelmente possuírem mais conhecimento dos medicamentos que consomem. Um estudo realizado por Abraão et al. $^{20}$ apresentou resultados similares ao presente estudo no qual a maioria dos participantes (92\%) relataram desconhecer qualquer tipo dessas reações, diferente do resultado encontrado por Rankel et al. ${ }^{1}$ no qual $40 \%$ não tinham conhecimento dos efeitos do uso abusivo de medicação.

No presente estudo, aproximadamente metade dos participantes afirmaram ler a bula dos medicamentos, sendo o sexo feminino mais prevalente o que pode ser devido ao fato das mulheres se cuidarem mais quanto ao uso dos fármacos. Este valor foi muito diferente do encontrado no estudo de Santana $^{7}$ na qual apenas $10 \%$ afirmaram ler a bula de medicamentos. A bula é um importante instrumento para informar o usuário quanto ao uso do medicamento, efeitos adversos, podendo gerar uma sensação superficial de domínio do saber médico, e mesmo com grande número de informações contidas nas bulas, as pessoas continuam a praticar a automedicação ${ }^{21}$. 
Dentre as justificativas para a realização da automedicação, as que mais foram relatadas pelos participantes foram: utilização de um fármaco anteriormente já prescrito pelo médico. Valor parecido corrobora com o estudo de Silva e Rodrigues ${ }^{21}$ no qual $53,1 \%$ dos participantes afirmaram que já se basearam em receitas médicas anteriores para tratar sintomas e doenças e $38,2 \%$ dos participantes afirmaram ter sido feita por indicação familiar. Já no estudo de Silva et al. ${ }^{2}, 30 \%$ dos participantes afirmaram usar prescrição médica antiga, e 30\% indicações de amigos e parentes.

Com relação à orientação do farmacêutico a maioria dos participantes afirmaram não receber orientações desse profissional, divergindo do estudo de Silva et al. ${ }^{22}$ que encontraram $56,84 \%$ dos entrevistados dizendo ser orientados pelo farmacêutico somente quanto à forma de utilização, e o restante, $43,16 \%$, responderam que não são orientados. $O$ farmacêutico tem papel fundamental na etapa de orientação da população para o uso correto de medicamentos, pois além de seus conhecimentos generalistas obtidos em sua formação, eles são os responsáveis pela orientação e dispensação segura. O trabalho da atenção farmacêutica junto a população no momento da dispensação do medicamento é de grande relevância, pois é nesse momento em que o paciente vai receber as orientações sobre como usar o medicamento, a dose correta, o tempo de tratamento, riscos ou benefícios, ou dependendo do caso sendo orientados a procurar uma unidade básica de saúde ${ }^{16}$. Concluindo a prática da automedicação se mostrou muito frequente na cidade estudada, principalmente entre o sexo feminino, evidenciando uma necessidade de maior conscientização da população, por meio de campanhas, reforçando a necessidade da atuação do farmacêutico, sendo ele o profissional final do ciclo do medicamento e o responsável pela dispensação do mesmo, momento essencial para orientação da população. O papel exercido pelo médico de orientador também é essencial na prevenção e controle da automedicação, sendo mais efetivo se ambos os profissionais (médico e farmacêutico) trabalhassem em conjunto.

\section{Conflitos de Interesse}

Os autores declaram não haver qualquer potencial de conflito de interesse que possa interferir na imparcialidade deste trabalho científico.

\section{REFERÊNCIAS}

1. Rankel SAO, Sato MDO, Santiago RM. Uso irracional dos anti-inflamatórios não esteroidais no município de Tijucas do Sul. Visão Acadêmica, v. 17, n. 4, 2017. DOI: http://dx.doi.org/10.5380/acd.v17i4.50205

2. Silva JAC, Gomes AL, Oliveira JPS, Sasaki YA, Maia BTB, Abreu BM. Prevalência de automedicação e os fatores associados entre os usuários de um Centro de Saúde Universitário. Rev Bras Clin Med, v. 11, n. 1, p. 27-30, 2013.

3. Burke A, Smyth E, Fitzgerald GA. Analgésicos Antipiréticos; Farmacoterapia da gota. In: Brunton LL, Chabner BA, Knollmann BC. Goodman \& Gilman: As Bases Farmacológicas da Terapêutica, $12^{a}$ edição. Porto Alegre. Mc Graw-Hill/ Art Med, 01/2012, p. 601-638.

4. Aquino DS. Por que o uso racional de medicamentos deve ser uma prioridade? Ciência \& Saúde Coletiva, v. 13, p. 733-736, 2008.

5. Pilger MC, Dombrowski G, Rebelo M, Tomasi E. Automedicação entre acadêmicos de medicina das Universidades Católica e Federal de Pelotas/RS. Revista da AMRIGS, Porto Alegre, 60 (1): xx-xx, jan-mar. 2016.

6. Da Silva FM, Goulart FC, Lazarini CA. Caracterização da prática de automedicação e fatores associados entre universitários do curso de Enfermagem. Rev. Eletr. Enf., 2014 jul/set; 16(3): 644-51. DOI: http://dx.doi.org/10.5216/ree.v16i3.20850

7. Santana HMM. Consumo de antiinflamatórios não esteroides em uma farmácia comunitária em Juazeiro do Norte-CE, Monografia (Especialização em Assistência Farmacêutica) - Escola de Saúde Pública do Ceará. Juazeiro do Norte, 2006.

8. Almeida-Junior G, Kamonseki DH, RostelatoFerreira S. Perfil de automedicação no município de São Miguel Arcanjo/SP. Espaço para a Saúde- 
Revista de Saúde Pública do Paraná, v. 17, n. 2, p. 93-100, 2016. DOI: $\quad$ http://dx.doi.org/10.22421/1517$\underline{7130.2016 v 17 n 2 p 93}$

9. Souza JFR, Marinho CLC, Guilam MCR. Consumo de medicamentos e internet: análise crítica de uma comunidade virtual. Revista da Associação de Medicina Brasileira. Rio de Janeiro, v. 54, n. 3, p. 225-231, 2008. DOI: http://dx.doi.org/10.1590/S0104$\underline{42302008000300015}$

10.Mengue SS, Dâmaso AB, Leão TNU, Silva DPT, Auxiliadora OM, Dourado APS et al. Prevalência da automedicação no Brasil e fatores associados. Revista de saúde pública. São Paulo. Vol. 50, supl. 2 (2016), p. 1s-11s., 2016. DOI: $\quad$ http://dx.doi.org/10.1590/S1518$\underline{8787.2016050006117}$

11.Karyna HOSD, Almeida FS, Jorge RSR. Estudo sobre automedicação no uso de antiinflamatórios não Esteroides na cidade de Valparaíso de Goiás. Revista Saúde e Desenvolvimento, v. 9, n. 5, p. 142-153, 2016.

12.Fontanella FG, Galato D, Remor KVT. Perfil de automedicação em universitários dos cursos da área da saúde em uma instituição de ensino superior do sul do Brasil. Rev. Bras. Farm, v. 94, n. 2, p. 154-160, 2013.

13. Lopes WFL, Coelho MROM, Oliveira JP, Araujo YMO, Melo MCN, Tapety FI. A prática da automedicação entre estudantes de uma instituição de ensino superior de TeresinaPI. Revista Interdisciplinar, v. 7, n. 1, p. 17-24, 2014.

14. Karen A, Woodfork, Knox Van Dyke. Agentes Antiinflamatorios e Anti-Reumaticos. In: Craig CR, Stitzel RE. Farmacologia Moderna com Aplicaçoes clinicas. $6^{a}$ Edição. Rio de Janeiro. Ed Guanabara Koogan S.A. 2005, p.398-413.

15. Carvalho WA. Anti-inflamatórios Não Esteroides, Analgésicos, Antipiréticos e Drogas Utilizadas no Tratamento da Gota. In: PENILDON, S. Farmacologia, $8^{a}$ Edição. Rio de Janeiro. Ed. Guanabara Koogan, 2012, p.440466. no Brasil e a importância do farmacêutico na orientação do uso racional de medicamentos de venda livre: uma revisão. Revista da Graduação, v. 9, n. 2, 2016.

17. Danieli $P$, Leal MB. Avaliação da segurança da dipirona: uma revisão. Rev. Bras. Farm., v. 84, n. (1), p. 17-20, 2003.

18. Ferreira $A L$, Rocha $C P$, Vieira $L M$, Dusse LMSA, Rezende D, Junqueira G, Carvalho MG. Alterações hematológicas induzidas por medicamentos convencionais e alternativos. Rev. Bras. Farm, v. 94, n. 2, p. 94101, 2013.

19. Oliveira GG. Painel Internacional de avaliação da segurança da Dipirona [Internacional Panel for the Evaluation of the Safety of Dipyrone]. Di.rio Oficial da Unio, Sec. I, p. 201-02, 16.08, 2001.

20. Abraão LM, Simas, JMM, Miguel TLB. Incidência da Automedicação e Uso Indiscriminado de Medicamentos entre Jovens Universitários. [dissertação]. Lins: Centro Universitário UniSalesiano, 2009.

21. Silva LAF, Rodrigues AMS. Automedicação entre estudantes de cursos da área de saúde, Rev. Bras. Farm. 95 (3), 961 - 975, 2014.

22. Silva DVR, Evangelista WD, Mota HF, Mota $B C F$, Royo VA. Automedicação e atenção farmacêutica sobre analgésicos em drogaria de Montes Claros-MG. Revista Multitexto, v. 2, n. 1, p. 45-49, 2013.

16. Soterio KA, Santos MA. A automedicação 